\title{
Pisa Syndrome in Parkinson's Disease: Evidence for Bilateral Vestibulospinal Dysfunction
}

\author{
Giulia Di Lazzaro,, ${ }^{1}$ Tommaso Schirinzi, ${ }^{1}$ Maria Pia Giambrone, ${ }^{1}$ Roberta Di Mauro ${ }^{10},{ }^{2}$ \\ Maria Giuseppina Palmieri, ${ }^{1}$ Camilla Rocchi, ${ }^{1}$ Michele Tinazzi, ${ }^{3}$ Nicola Biagio Mercuri, ${ }^{1,4}$ \\ Stefano Di Girolamo, ${ }^{2}$ and Antonio Pisani ${ }^{1,4}$ \\ ${ }^{1}$ Department of Systems Medicine, University of Rome Tor Vergata, Rome, Italy \\ ${ }^{2}$ Department of Otorhinolaryngology, University of Rome Tor Vergata, Rome, Italy \\ ${ }^{3}$ Department of Neuroscience, Biomedicine and Movement Sciences, University of Verona, Verona, Italy \\ ${ }^{4}$ Fondazione Santa Lucia IRCCS, Rome, Italy \\ Correspondence should be addressed to Antonio Pisani; pisani@uniroma2.it
}

Received 14 June 2018; Revised 30 August 2018; Accepted 24 September 2018; Published 15 October 2018

Academic Editor: Pablo Mir

Copyright (c) 2018 Giulia Di Lazzaro et al. This is an open access article distributed under the Creative Commons Attribution License, which permits unrestricted use, distribution, and reproduction in any medium, provided the original work is properly cited.

\begin{abstract}
Introduction. Pisa syndrome (PS) is a postural complication of Parkinson's disease (PD). Yet, its pathophysiology remains unclear, although a multifactorial component is probable. Cervical vestibular evoked myogenic potentials (cVEMPs) explore vestibulospinal pathway, but they have not been measured yet in PD patients with PS (PDPS) to assess a potential vestibular impairment. Materials and Methods. We enrolled 15 PD patients, 15 PDPS patients, and 30 healthy controls (HCs). They underwent neurological examination and were examined with Unified Parkinson's Disease Rating Scale II-III (UPDRSII-III), audiovestibular workup, and cVEMP recordings. Data were analysed with Chi-square, one-way ANOVA, multinomial regression, nonparametric, and Spearman's tests. Results. cVEMPs were significantly impaired in both PD and PDPS compared with HCs. PDPS exhibited more severe cVEMP abnormalities with prevalent bilateral loss of potentials, compared with the PD group, in which a prevalent unilateral loss was instead observed. No clinical-neurophysiological correlations emerged. Conclusions. Differently from HC, cVEMPs are altered in PD. Severity of cVEMPs alterations increases from PD without PS to PDPS, suggesting an involvement of vestibulospinal pathway in the pathophysiology of PS. Our results provide evidence for a significant impairment of cVEMPs in PDPS patients and encourage further studies to test validity of cVEMPs as diagnostic and prognostic biomarkers of PD progression.
\end{abstract}

\section{Introduction}

Parkinson's disease (PD) is a common neurodegenerative disease characterized by both motor and nonmotor features. While motor signs (tremor, rigidity, and bradykinesia) mainly originate from the loss of dopaminergic neurons of substantia nigra pars compacta (SNpc) $[1,2]$, the pathophysiology of the large cohort of symptoms complicating the disease course, such as postural impairment, remains unclear, although it has been ascribed to the progressive degeneration of other cortical and subcortical structures [1].
Pisa syndrome (PS) is a peculiar postural complication, consisting in a persistent lateral trunk flexion (LTF) of more than $10^{\circ}$ that can be reduced by passive mobilization or supine positioning, occurring throughout the disease course but favoured by the longer disease duration and responsible for severe disability [3-5]. Neurochemical imbalance in the basal ganglia homeostasis, defective sensory-motor integration, abnormal body scheme perception, together with a peripheral involvement consisting in myopathy, and other musculoskeletal affections have been proposed to contribute to the pathophysiology of PS $[3,6,7]$. However, the precise mechanisms have not been clarified yet. 
Recently, a renewed interest emerged for vestibular evoked myogenic potentials (VEMPs), utilized for testing short-latency myogenic responses evoked by sound stimulation of vestibular system [8]. Specifically, cervical vestibular evoked myogenic potentials (cVEMPs) represent the sternocleidomastoid (SCM) muscle response to sound activation of saccule and signal transmission via the vestibulospinal tract. Thus, cVEMPs are a simple and reliable tool to explore the activity of the vestibulocollic reflex, linking the VIII and XI cranial nuclei, thereby representing an index of brainstem integrity [9-12]. Indeed, the vestibular system has been involved in the pathophysiology of PS [13], as well as in falls and other motor complications of advanced PD [13-15].

In spite of such evidence, to date, few studies addressed these issues in PD population. Evidence has been provided for the loss of muscular potentials, in the absence of clear correlations with relevant clinical features, such as disease duration and severity, gait, or sleep disturbances [15-17]. However, cVEMPs measurement has not been utilized yet to evaluate vestibular involvement in PS.

In this study, we recorded cVEMPs to assess vestibular integrity in PD patients with PS (PDPS) compared with both PD patients without PS and healthy controls (HCs).

\section{Materials and Methods}

2.1. Materials. A total of 60 subjects were consecutively recruited at the movement disorders outpatient service, Tor Vergata University Hospital, Rome, Italy, between 2015 and 2017. Subjects were divided into 3 groups: PD patients $(n=15)$, PD patients with PS (PDPS, $n=15)$, and healthy controls (HC, $n=30)$. Idiopathic $\mathrm{PD}$ was diagnosed according to the British Parkinson's Disease Society Brain Bank criteria and confirmed by DaTscan [18]; PDPS patients showed trunk lateral flexion of at least $10^{\circ}$ measured with a wall goniometer, alleviated by passive mobilization or supine positioning, according to recent diagnostic criteria [3]. HCs were healthy age-matched control subjects recruited from nonblood relatives of PD patients who did not show any neurological sign.

Exclusion criteria included dementia (MMSE score $<24$ ), cervical herniation, history of orthopaedic diseases (such as scoliosis), major spinal surgery, improper neck movements that could alter audiological assessment, middle ear diseases, hearing thresholds exceeding $50 \mathrm{dBHL}$, and medical therapy potentially interfering with vestibular function or potentially able to induce PS (e.g., neuroleptics).

All patients were evaluated by a movement disorder specialist, with Unified Parkinson's Disease Rating Scale (UPDRS) Sections II and III and Hoehn and Yahr scale (H \& Y). Medical therapy was accurately recorded, and total levodopa equivalent daily dose (LEDD) was calculated for each patient.

All enrolled subjects underwent audiological workup to exclude hearing impairment, which could alter VEMPs recording. Then, cVEMPs were recorded, as described below. The entire assessment was performed in "on-therapy" state.
The study was conducted in agreement with ethical principles of Helsinki declaration. Informed consent was obtained from all participants.

\subsection{Procedures}

2.2.1. cVEMPs Recording. The test was performed in a hypoechoic room, with the patient lying down on an examination bed, head positioned at $30^{\circ}$. Five surface electrodes were placed, the negative ones on the medium third of SCM muscles bilaterally, the positive ones in the centre of claviculae, and the ground electrode on the sternum. While maintaining tonic contraction of SCM, a bilateral acoustic stimulation (alternate click) was given to the subject continuously for $30 \mathrm{~s}$, at a frequency of $4 \mathrm{~Hz}$, duration of $100 \mu \mathrm{s}$ and at an intensity of $135 \mathrm{~dB}$ SPL, similarly in the three groups. The compound muscle action potentials (CMAPs) were then recorded as described [9].

Although muscle tonic activation was not measured, it was continuously monitored by visual feedback to assist the subject in maintaining the task. The recorded signals were amplified, averaged, and band-pass filtered $(10-500 \mathrm{~Hz})$. The procedure was performed at least twice to ensure reproducibility; grand average was analysed. CMAPs were evaluated qualitatively (present/absent) and quantitatively, measuring wave latencies and peak amplitudes from stimulus onset to the peak of the initial positivity (P13) and subsequent negativity (N23) [9]; interpeak latencies were also calculated [11] (Figure 1).

2.2.2. Audiological Workup. Each subject underwent an otolaryngologic evaluation, including otoscopic examination, acoustic impedance test and pure-tone audiometry (PTA) to exclude middle ear disorders. Hearing loss was estimated bilaterally and for each pure-tone frequency stimulation (from 125 to $8000 \mathrm{~Hz}$ ). The intensity threshold of the acoustic reflex was determined for each ear using $500 \mathrm{~Hz}, 1000 \mathrm{~Hz}, 2000 \mathrm{~Hz}$, and $4000 \mathrm{~Hz}$ stimulus tones. The stimulus was presented either to the same ear as the compliance probe (ipsilateral reflex) or to the opposite ear (contralateral reflex).

For the Brainstem Auditory Evoked Responses (BAER) assessment, the active electrode was mounted to the middle of the forehead "Fpz," the reference electrode to the ipsilateral mastoid "M1," and the ground to the contralateral one "M2." The test procedures followed the Sininger protocol [19]. Analysis of BAER was done quantitatively to assess the absolute latencies of waves I, III, and V and interpeak latencies of these waves (I-III, III-V, and I-V). Qualitative analysis of the waveform morphology included the subjective judgment on the shape and the quality of the waveforms.

2.3. Statistical Analysis. Differences among groups in categorical variables were calculated using the Chi-square test. Audiometric data were assessed separately by KruskalWallis test for multiple comparisons and Wilcoxon test for repeated measures, as they were nonnormally distributed. 


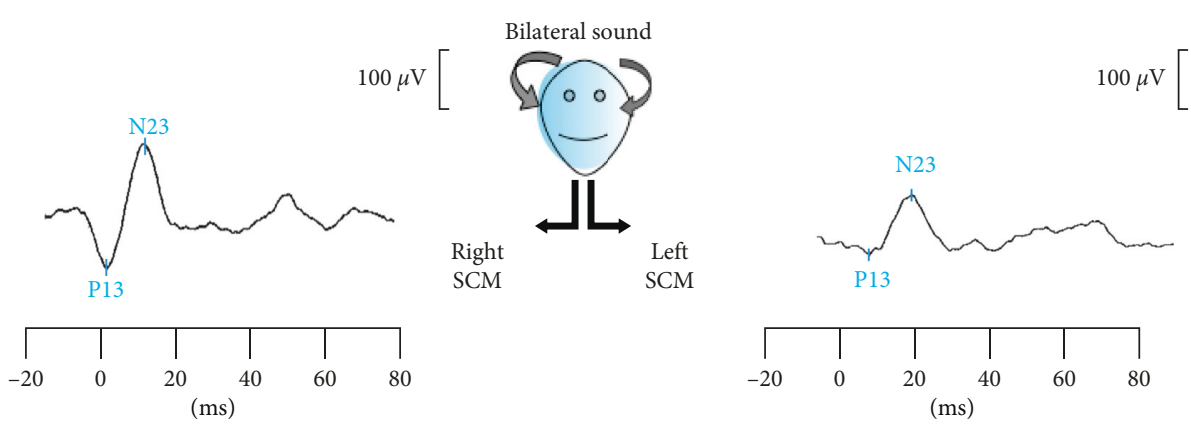

(a)
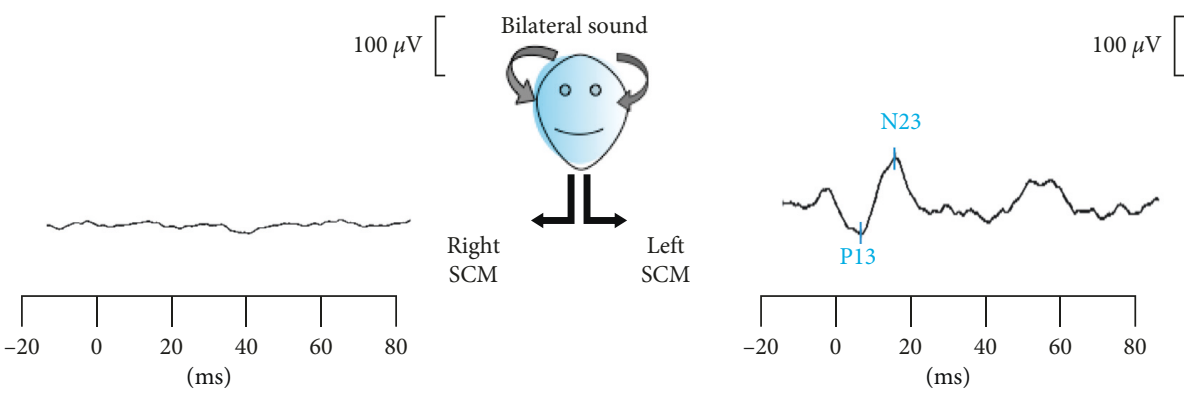

(b)

Figure 1: cVEMP recording in normal (a) and altered (b) conditions. In both (a) and (b) boxes, the two traces correspond, respectively, to right and left SCM muscle potential evoked by bilateral sound stimulation. P13 represents the potential's first peak of positivity, N23 the subsequent peak of negativity. In (a), cVEMPs are present bilaterally; in (b), cVEMP is lost on the right side.

The one-way ANOVA test with Bonferroni correction was performed for cVEMPs amplitude and latencies analysis among groups.

Correlations between neurophysiological parameters (unilateral or bilateral absence of cVEMPs, their amplitudes and latencies) and clinical features (disease duration, age of onset, UPDRS II-III as total scores and single subitems, degrees of LTF, and bending side) were assessed with Spearman's test. Moreover, after testing differences in cVEMPs alterations within the groups, the association between vestibular dysfunction and clinical presentation of PD was further assessed by means of multinomial logistic regression, using disease duration as covariate.

The level of statistical significance ( $p$ value) was set at 0.05 . Statistical analyses were performed using the SPSS version 21 for Mac (SPSS Inc., Chicago).

\section{Results}

Main demographic, clinical, and neurophysiological data are summarized in Table 1.

Groups did not significantly differ in age, gender, and auditory testing (auditory thresholds and BAER latencies, data not shown). Disease duration, UPDRS part II-III scores, and LEDD were significantly higher in PDPS than in PD (respectively, $p<0.001, p<0.001, p<0.001$, and $p=0.013$ ). Conversely, no difference emerged in the prevalence of dopaminergic medications used in PD and PDPS groups; specifically, 11/15 PD patients and 9/15 PDPS patients were on dopamine agonists, 10/15 PD and 9/15 PDPS were on
MAO-B inhibitors, and 12/15 PD and 13/15 PDPS were on levodopa.

Consistent with previous findings, the main alteration we observed in cVEMPs within the PD groups was the unilateral or bilateral absence of evoked potentials (Figure 1); even if PDPS and PD patients had longer P13 and N23 latencies, no significant differences emerged among other analysed variables.

Table 1 shows, for each group, the percentage of "normal," "unilateral absence," and "bilateral absence" of cVEMPs. Chi-square analysis demonstrated that normal cVEMPs were significantly reduced in both PD and PDPS compared with HC (respectively, $p<0.001$ and $p<0.001$ ). However, the pattern of cVEMP abnormalities significantly differed between PDPS and PD $(p<0.0001)$, with PDPS showing lower normal responses $(p<0.05)$ and more frequent "bilateral absence" $(p<0.001)$; otherwise "unilateral absence" was more common in PD than in PDPS $(p<0.05)$ (Figure 2).

Multinomial logistic regression showed that "unilateral absence" of cVEMPs but not "bilateral absence" is significantly associated with pure PD condition $(T=21.9$, $p<0.001)$, independently from disease duration. Moreover, either "unilateral" or "bilateral" cVEMPs absence are directly associated with PDPS condition (respectively, $T=3.4, p=$ $0.02 p=0.02$ and $T=21.2, p<0.001)$, as well as disease duration $(T=0.4, p=0.004)$.

Finally, correlation analysis between neurophysiological and clinical parameters did not provide any significant result among the groups. 
TABLE 1: Clinical and electrophysiological data ( $\mathrm{y}=$ years; $\mathrm{M}=$ males; $\mathrm{F}=$ females; $\mathrm{mg}=$ milligrams; $\mathrm{ms}=\mathrm{millisecond}$; $\mathrm{mcV}=\mathrm{microVolt}$; $\mathrm{LTF}=$ lateral trunk flexion; $\mathrm{R}=$ right; $\mathrm{L}=$ left; $\mathrm{LEDD}=$ levodopa equivalent daily dose; $\mathrm{n} . \mathrm{s}$. = statistically nonsignificant). Data are expressed as mean \pm standard deviation (SD).

\begin{tabular}{|c|c|c|c|c|}
\hline & PDPS $(n=15)$ & $\mathrm{PD}(n=15)$ & $\mathrm{HC}(n=30)$ & \\
\hline Age (y) & $73.3 \pm 3.6$ & $69.6 \pm 7.11$ & $69.36 \pm 6.67$ & n.s. \\
\hline Gender $(\mathrm{M} / \mathrm{F})$ & $8 / 7$ & $7 / 8$ & $15 / 15$ & n.s. \\
\hline Disease duration $(\mathrm{y})$ & $8.7 \pm 4.4$ & $4.6 \pm 3.83$ & - & $p<0.001$ \\
\hline UPDRS II & $12.4 \pm 2.8$ & $6.2 \pm 3$ & - & $p<0.001$ \\
\hline UPDRS III & $30 \pm 6.1$ & $18.9 \pm 6.1$ & - & $p<0.001$ \\
\hline $\operatorname{LTF}\left({ }^{\circ}\right)$ & $21 \pm 9$ & - & - & \\
\hline Side of $\operatorname{LTF}(\mathrm{R} / \mathrm{L})$ & $7 / 8$ & - & - & \\
\hline LEDD (mg) & $814 \pm 275$ & $369 \pm 202$ & - & $p=0.013$ \\
\hline Normal cVEMPs & $48 \%$ & $68 \%$ & $97 \%$ & $p<0.001$ \\
\hline Unilateral absence of cVEMPs & $12 \%$ & $26 \%$ & $0 \%$ & $p<0.001$ \\
\hline Bilateral absence of cVEMPs & $40 \%$ & $6 \%$ & $3 \%$ & $p<0.001$ \\
\hline P13 latencies (ms) & $17.8 \pm 10.82$ & $14.32 \pm 3.3$ & $16.07 \pm 8.97$ & n.s. \\
\hline N23 latencies (ms) & $26.53 \pm 10.28$ & $22.68 \pm 4.2$ & $25.77 \pm 10.48$ & n.s. \\
\hline P13 amplitudes (mcV) & $46.18 \pm 29.88$ & $41.92 \pm 37.75$ & $40.57 \pm 26.1$ & n.s. \\
\hline N23 amplitudes (mcV) & $-44.9 \pm 35.26$ & $-54.6 \pm 34.01$ & $-63.16 \pm 42.31$ & n.s. \\
\hline Interpeak latencies (ms) & $8.6 \pm 2.5$ & $8.5 \pm 2.3$ & $9.6 \pm 3.4$ & n.s. \\
\hline
\end{tabular}

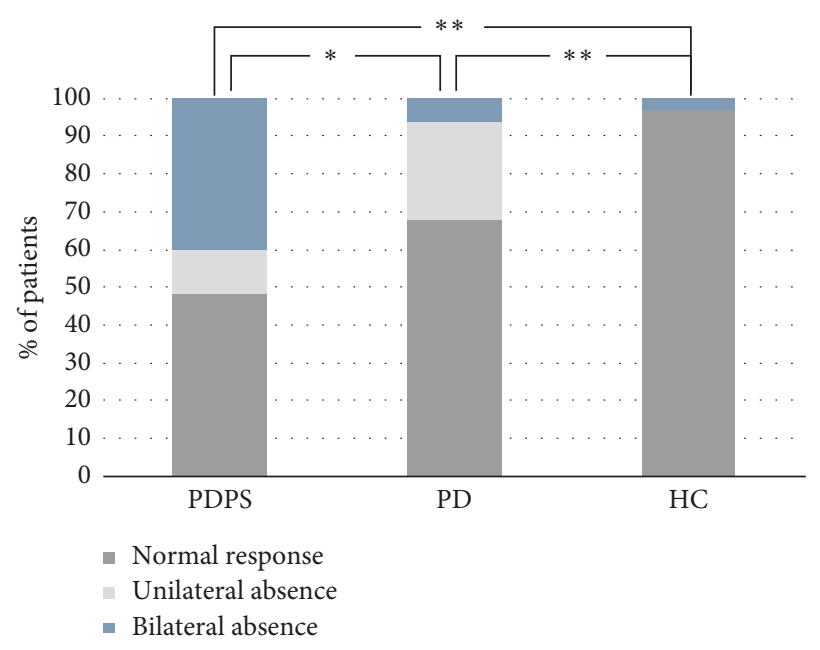

FIGURE 2: The plot summarizes the percentage of different cVEMPs responses in the three groups. Significance markers $\left(^{*}=p<0.05\right.$; $\left.{ }^{* *}=p<0.001\right)$ refer to the presence of "normal responses." Other data are expressed in the text.

\section{Discussion}

The neuropathological hallmark of PD is the ascending accumulation of $\alpha$-synuclein immunoreactive Lewy bodies (LB) from SNpc and other brainstem structures to superior brain areas; however, LB deposition has been recently demonstrated in all brainstem fiber tracts and cranial nerve nuclei, including the vestibular system [20]. Of note, dopamine, whose levels are critically reduced in $\mathrm{PD}$, is a fundamental modulator of vestibular nuclei function [21]. cVEMPs are now considered a reliable tool to assess vestibular and brainstem functions in distinct neurological disorders, including neuroaudiological, demyelinating and cerebrovascular diseases, migraine, and CNS tumours $[8,22]$. Conversely, few studies examined the potential utilization of cVEMPs as a tool to monitor neurodegenerative diseases, particularly PD [15].

In our cross-sectional analysis of cVEMPs among PDPS, PD patients without PS and HCs without neurodegenerative conditions, we demonstrate that PD patients, compared with the latter group, have significantly abnormal vestibular evoked responses, consisting in unilateral or bilateral loss of cVEMPs. Furthermore, our findings indicate that the neurophysiological profile differs between PD patients with and without PS. The PD group mostly exhibits the unilateral absence of cVEMPs, whereas the PDPS group shows prevalently bilateral loss of responses. Our data on vestibular dysfunction may thus reflect the progression of brainstem neurodegeneration along disease course, with a progressive worsening in advanced stages, when postural complications are more frequently observed [23].

Despite the absence of significant correlations between neurophysiological parameters and specific clinical features, our results show a peculiar alteration of vestibular functioning in PS. While a previous study demonstrated unilateral peripheral vestibular hypofunction, ipsilateral to the LTF side [13], we observed a higher prevalence of bilateral alterations in PDPS patients here. A note of caution in the interpretation of our data is required, as our study lacks peripheral testing, which might have been useful in the anatomoclinical correlation. However, combining existing data with our novel observations, we hypothesize that central vestibular impairment might concur with peripheral dysfunction in patients with PS [13], suggesting the specific frailty of the vestibular system in this condition, even if longer disease duration might also play a role.

The study also included an audiometric examination of the three groups, which showed no significant differences in the hearing thresholds of subjects with PD, PDPS, and HCs. This finding partially disagrees with evidence by Vitale and colleagues [24, 25], instead demonstrating the occurrence of neurosensory hearing impairment in PD patients. The exclusion, in our study, of subjects with hearing thresholds 
over $50 \mathrm{dBHL}$ even at a single frequency and additionally the smaller sample size and the different clinical-demographic features of the cohorts may account for this discrepancy.

\section{Conclusions}

cVEMPs are a noninvasive and inexpensive test exploring vestibulospinal tract, a crucial pathway for postural control. Despite the small sample size, here we observed that vestibulospinal dysfunction progresses in PD, with a milder involvement (unilateral loss of cVEMPs) in the absence of postural disabilities and more severe alterations (bilateral loss of cVEMPs) in the presence of PS. Therefore, we suggest that a major impairment of vestibular system might be involved in pathophysiology of PS, probably mirroring more advanced brainstem pathology in parallel with a longer disease duration. Further prospective studies on larger samples are mandatory to understand the associations between audiovestibular dysfunction and clinical progression of PD, allowing to establish the potential usefulness of cVEMPs as diagnostic and prognostic biomarkers for PD.

\section{Data Availability}

The data used to support the findings of this study are included within the article.

\section{Disclosure}

The abstract has been presented at the 4th Congress of Accademia LIMPE-DISMOV in May 2018.

\section{Conflicts of Interest}

The authors declare no conflicts of interest.

\section{References}

[1] L. V. Kalia and A. E. Lang, "Parkinson's disease," The Lancet, vol. 386, no. 9996, pp. 896-912, 2015.

[2] T. Schirinzi, G. Madeo, G. Martella et al., "Early synaptic dysfunction in Parkinson's disease: insights from animal models," Movement Disorders, vol. 31, no. 6, pp. 802-813, 2016.

[3] M. Tinazzi, C. Geroin, M. Gandolfi et al., "Pisa syndrome in Parkinson's disease: an integrated approach from pathophysiology to management," Movement Disorders, vol. 31, no. 12, pp. 1785-1795, 2016.

[4] M. Tinazzi, A. Fasano, C. Geroin et al., "Pisa syndrome in Parkinson disease: an observational multicenter Italian study," Neurology, vol. 85, no. 20, pp. 1769-1779, 2015.

[5] M. Alwardat, G. Di Lazzaro, T. Schirinzi, P. Sinibaldi Salime, N. B. Mercuri, and A. Pisani, "Does pisa syndrome affect upper limb function in patients with Parkinson's disease? an observational cross-sectional study," NeuroRehabilitation, vol. 42, no. 2, pp. 143-148, 2018.

[6] K. M. Doherty, B. P. van de Warrenburg, M. C. Peralta et al., "Postural deformities in Parkinson's disease," The Lancet Neurology, vol. 10, no. 6, pp. 538-549, 2011.

[7] P. Barone, G. Santangelo, M. Amboni, M. T. Pellecchia, and C. Vitale, "Pisa syndrome in Parkinson's disease and parkinsonism: clinical features, pathophysiology, and treatment," Lancet Neurology, vol. 15, no. 10, pp. 1063-1074, 2016.

[8] T. D. Fife, J. G. Colebatch, K. A. Kerber et al., "Practice guideline: cervical and ocular vestibular evoked myogenic potential testing," Neurology, vol. 89, no. 22, pp. 2288-2296, 2017.

[9] J. G. Colebatch, G. M. Halmagyi, and N. F. Skuse, "Myogenic potentials generated by a click-evoked vestibulocollic reflex," Journal of Neurology, Neurosurgery and Psychiatry, vol. 57, no. 2, pp. 190-197, 1994.

[10] E. R. de Natale, F. Ginatempo, K. S. Paulus et al., "Paired neurophysiological and clinical study of the brainstem at different stages of Parkinson's disease," Clinical Neurophysiology, vol. 126, no. 10, pp. 1871-1878, 2015.

[11] S. M. Rosengren, M. S. Welgampola, and J. G. Colebatch, "Vestibular evoked myogenic potentials: past, present and future," Clinical Neurophysiology, vol. 121, no. 5, pp. 636-651, 2010.

[12] M. Pötter-Nerger, S. Govender, G. Deuschl, J. Volkmann, and J. G. Colebatch, "Selective changes of ocular vestibular myogenic potentials in Parkinson's disease," Movement Disorders, vol. 30, no. 4, pp. 584-589, 2015.

[13] C. Vitale, V. Marcelli, T. Furia et al., "Vestibular impairment and adaptive postural imbalance in parkinsonian patients with lateral trunk flexion," Movement Disorders, vol. 26, no. 8, pp. 1458-1463, 2011.

[14] P. A. Pahapill and A. M. Lozano, "The pedunculopontine nucleus and Parkinson's disease," Brain, vol. 123, no. 9, pp. 1767-1783, 2000.

[15] E. R. de Natale, F. Ginatempo, K. S. Paulus et al., "Abnormalities of vestibular-evoked myogenic potentials in idiopathic Parkinson's disease are associated with clinical evidence of brainstem involvement," Neurological Sciences, vol. 36, no. 6, pp. 995-1001, 2015.

[16] L. Pollak, T. Prohorov, M. Kushnir, and M. Rabey, "Vestibulocervical reflexes in idiopathic Parkinson disease," Neurophysiologie Clinique/Clinical Neurophysiology, vol. 39, no. 45, pp. 235-240, 2009.

[17] J. Venhovens, J. Meulstee, B. R. Bloem, W. I. M. Verhagen, and J. Foxe, "Neurovestibular analysis and falls in Parkinson's disease and atypical parkinsonism," European Journal of Neuroscience, vol. 43, no. 12, pp. 1636-1646, 2016.

[18] A. J. Hughes, S. E. Daniel, L. Kilford, and A. J. Lees, “Accuracy of clinical diagnosis of idiopathic Parkinson's disease: a clinico-pathological study of 100 cases," Journal of Neurology, Neurosurgery and Psychiatry, vol. 55, no. 3, pp. 181184, 1992.

[19] Y. S. Sininger, "Auditory brain stem response for objective measures of hearing," Ear and Hearing, vol. 14, no. 1, pp. 23-30, 1993.

[20] K. Seidel, J. Mahlke, S. Siswanto et al., "The brainstem pathologies of Parkinson's disease and dementia with lewy bodies," Brain Pathology, vol. 25, no. 2, pp. 121-135, 2015.

[21] P. F. Smith, "Age-related neurochemical changes in the vestibular nuclei," Frontiers in Neurology, vol. 7, pp. 1-9, 2016.

[22] J. Venhovens, J. Meulstee, and W. I. M. Verhagen, "Vestibular evoked myogenic potentials (VEMPs) in central neurological disorders," Clinical Neurophysiology, vol. 127, no. 1, pp. 40-49, 2016.

[23] H. Braak, E. Ghebremedhin, U. Rüb, H. Bratzke, and K. Del Tredici, "Stages in the development of Parkinson's diseaserelated pathology," Cell and Tissue Research, vol. 318, no. 1, pp. 121-134, 2004 
[24] C. Vitale, V. Marcelli, R. Allocca et al., "Hearing impairment in Parkinson's disease: expanding the nonmotor phenotype," Movement Disorders, vol. 27, no. 12, pp. 1530-1535, 2012.

[25] C. Vitale, V. Marcelli, T. Abate et al., "Speech discrimination is impaired in parkinsonian patients: expanding the audiologic findings of Parkinson's disease," Parkinsonism and Related Disorders, vol. 22, pp. S138-S143, 2016. 




The Scientific World Journal
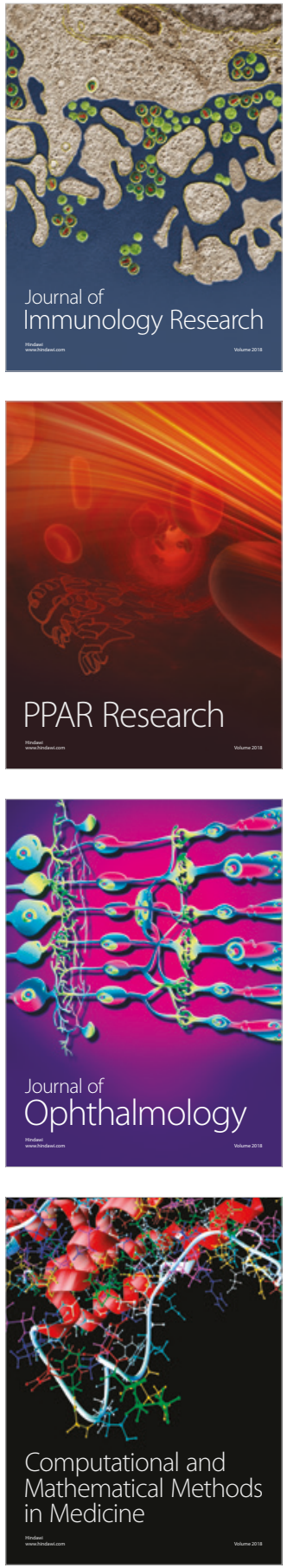

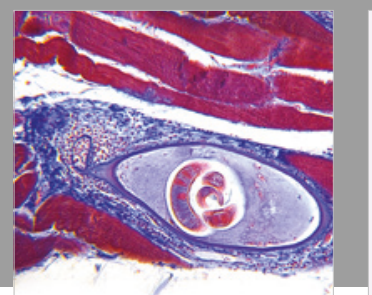

Gastroenterology Research and Practice

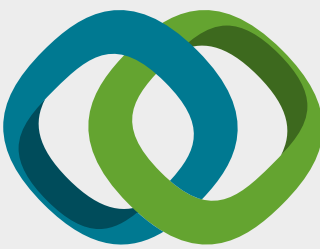

\section{Hindawi}

Submit your manuscripts at

www.hindawi.com
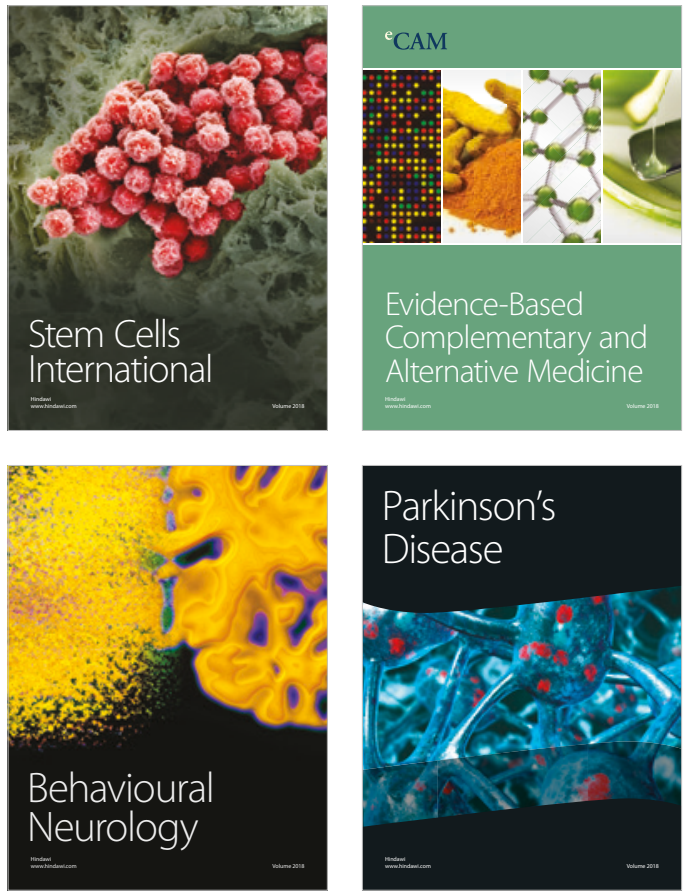

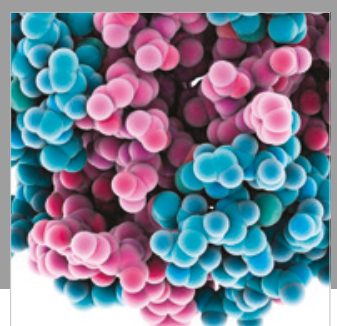

ournal of

Diabetes Research

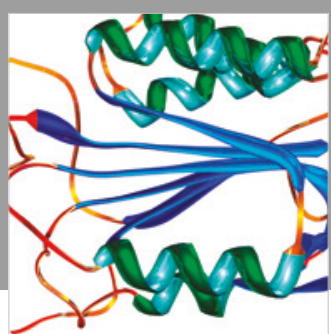

Disease Markers
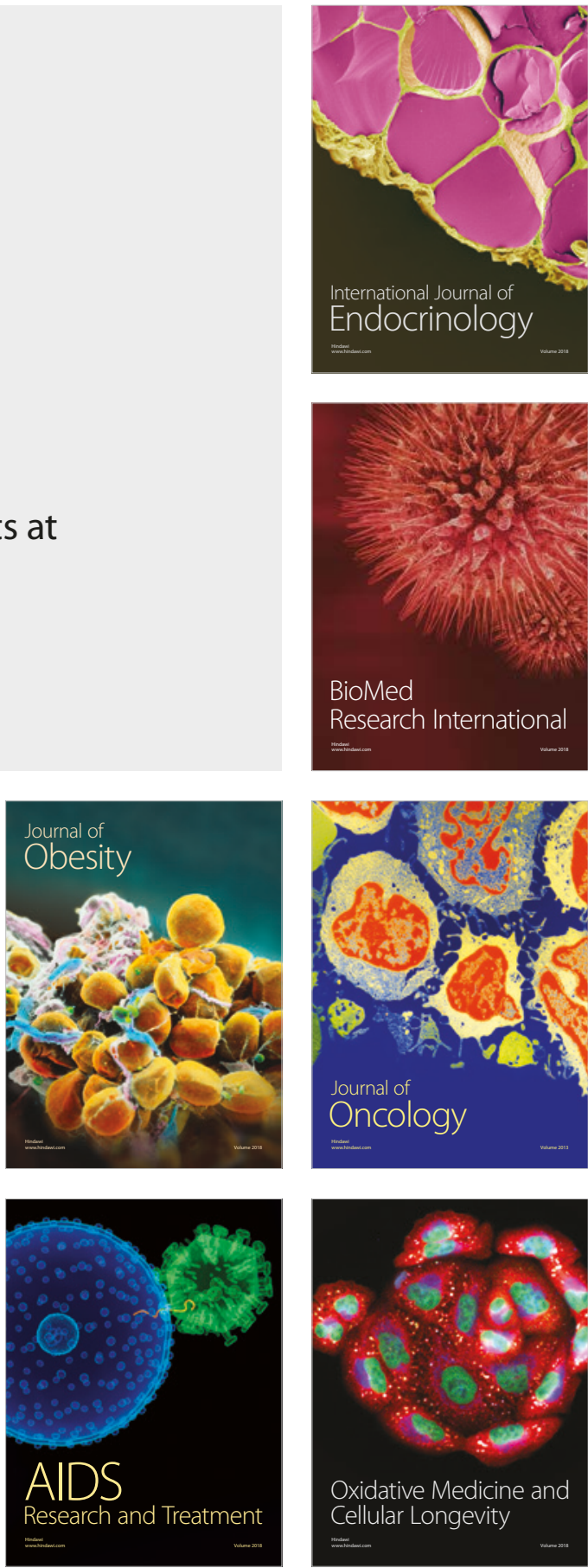